\title{
The Hydraulic Infinite Linear Actuator for Efficient and Flexible Timber and Agricultural Logistics
}

\author{
Magnus Landberg, Magnus Sethson*, Petter Krus* \\ Landberg Solutions AB, Linköping, Sweden \\ E-mail: magnus.landberg@saabgroup.com, magnus.sethson@liu.se, petter.krus@liu.se \\ * Division of Fluid and Mechatronic Systems; \\ Department of Management and Engineering, \\ Linköping University, Linköping, Sweden
}

\begin{abstract}
In forestry and agriculture industry, robust and power-dense hydraulics have long played an important role for a rational and cost-effective logistics. In these fields there is a trend towards longer, larger and heavier vehicles and machines. Within the forestry business there is a need to develop transport vehicles with lower energy consumption. This can be done by improving the vehicles' aerodynamics. The air resistance of unloaded timber trucks and timber trains will be significantly reduced if stakes and banks are put together. Furthermore, there is a need to place banks and stakes at individual distances individually to accomplish different load combinations. In agriculture when sowing, it is an advantage to be able to flexibly position row units on a seed drill in optimal distances from each other to secure productive and sustainable farming. However, most present seed drills have fixed row distances and thus have a low adaptability for different crops or soil conditions. There is also a need for a faster transport of the seed drill between different fields.

Future actuation systems for forestry and agricultural vehicles and machines can be improved by utilizing a new sort of hydraulic linear actuator technology, the Hydraulic Infinite Linear Actuator (HILA). HILA technology also has an impact on heavy and dangerous manual steps when changing banks and stakes on timber vehicles. The adjustment can be controlled from the cabin, thus eliminating manual steps. Heavy bank elements on a timber vehicle can be positioned individually with high locking force. In the agricultural context, it is possible to quickly change between different inter-row spacing on a seed drill when using HILA technology, enabling a multi-purpose seed drill and inter-row cultivator. HILA long stroke capability also facilitates a smooth folding into a compact transport position. With the bills are gathered, a low center of gravity will be accomplished. This enables a more stable vehicle dynamics and enables a higher speed.
\end{abstract}

HILA is based on a well-known hydraulic clamping element technology, where the piston and the piston rod can be coupled and uncoupled by means of the clamping element. The HILA invention, in its simplest usage, provides new features to hydraulic cylinders, such as providing very long strokes and small chamber volumes, which means high stiffness and low capacitance. However, the invention also enables lower weight and volume of the actuator when compared to conventional hydraulic cylinders. This is a new way to generate and distribute mechanical linear movement and force by using hydraulic actuators in a cost effective way. The technology also represents a new sort of digital hydraulics. The technology is best suited for relatively slow dynamics and where the movement pattern is well-known.

Keywords: hydraulic actuator, incremental control, digital hydraulics, logistics

\section{Introduction to HILA technology}

The HILA technology combines two short-stroke cylinders with two engaging and disengaging clamping mechanisms into one actuator with long stroke length. The clamping mechanism, a sort of hydraulic membrane, is connecting the piston and the rod, when pressurised, see fig. 1. The friction between the clamping mechanism and the piston rod is $\mu=0.1$ [3]. Spiral grooves in the clamping mechanism ensure that excess oil is removed in order to maintain the friction [3]. By using simple logic valves for pressurizing, the piston and rod can be connected and disconnected with a maximum secure and reliable clamping action in a fast way. The motion of each single shortstroke piston linked together by the clamping mechanisms creates the motion of the piston rod. For movements, one of the pistons is connected alternatively to the rod providing the drive. In this way the two pistons are moving the rod alternatingly in a kind of rope climbing motion. 


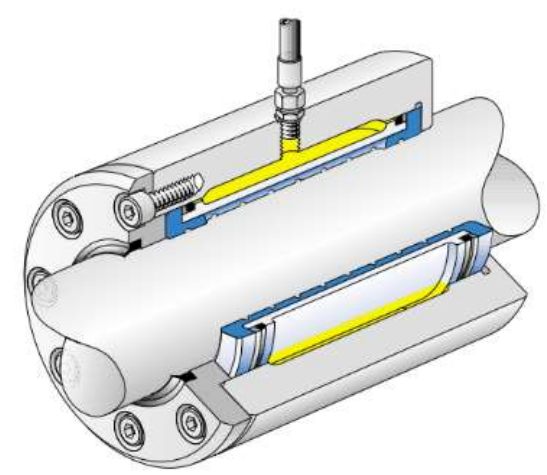

Figure 1. The clamping mechanism built into a hub [3].

In fig. 2 and 3 the actuation process of the cylinders is described. After step 7 the process will continue with step 2 (see also https://www.youtube.com/watch? $\mathrm{v}=\mathrm{tVJkqC} 2 \mathrm{w} 5 \mathrm{ws}$ ).

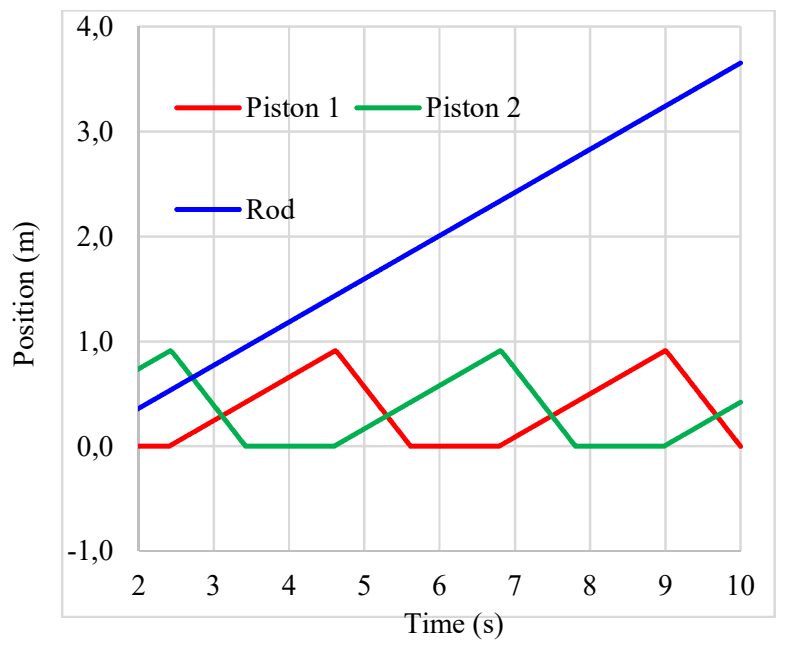

Figure 2. The positions of the pistons and piston rod during motion.

The actuator is the whole mechanism which is driving the load. It includes the piston rod, the hydraulic cylinders and its clamping elements and the position sensors. The shift of the load from one cylinder to the other is called load shift.
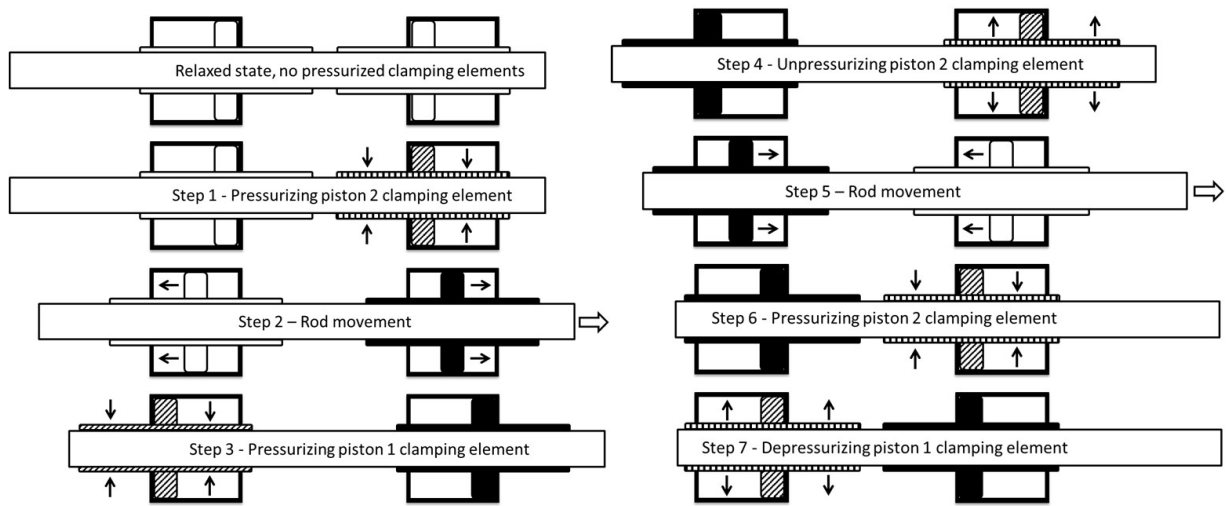

Figure 3. The actuation process of the cylinders. 
The pressurization of the clamping element and thus the engaging can be implemented by a separate port through the piston (see fig. 4) and using a separate $3 / 2$ valve. The purpose is to pressurise the small membrane volume and a minimal flow is needed. Thus a small and fast operating valve can be used. The piston and the clamping element are independently controlled by a timing procedure.

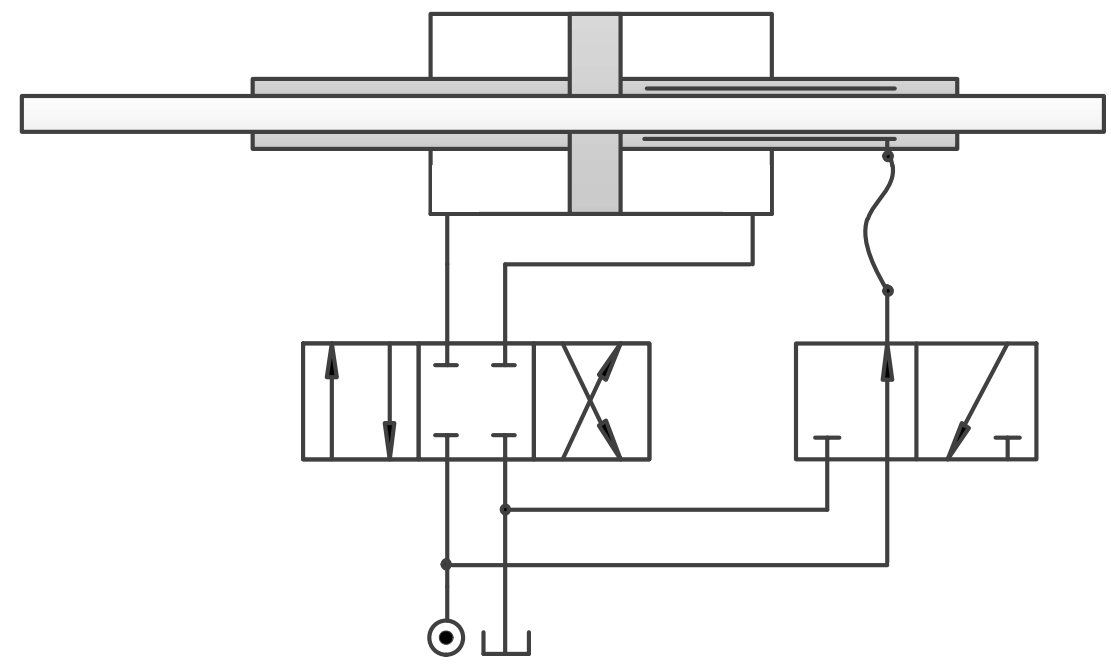

Figure 4. Separate pressurization of the clamping element.

In comparison with conventional long-stroke hydraulic actuators, HILA can offer shorter, more compact and easier-to-place solutions, especially on mobile vehicles and machines. Since the piston areas in the cylinders are symmetrical, marginal variation of the volume of oil in the system is obtained and a much smaller oil reservoir is required (see fig. 5). A challenge with this technology is to obtain a linear motion with a relatively low pulsation level. In many applications, however, smaller pulsations are acceptable. The technology is best suited for mobile applications with relatively slow dynamics and where the movement pattern is largely kinematic and well-known at beforehand.

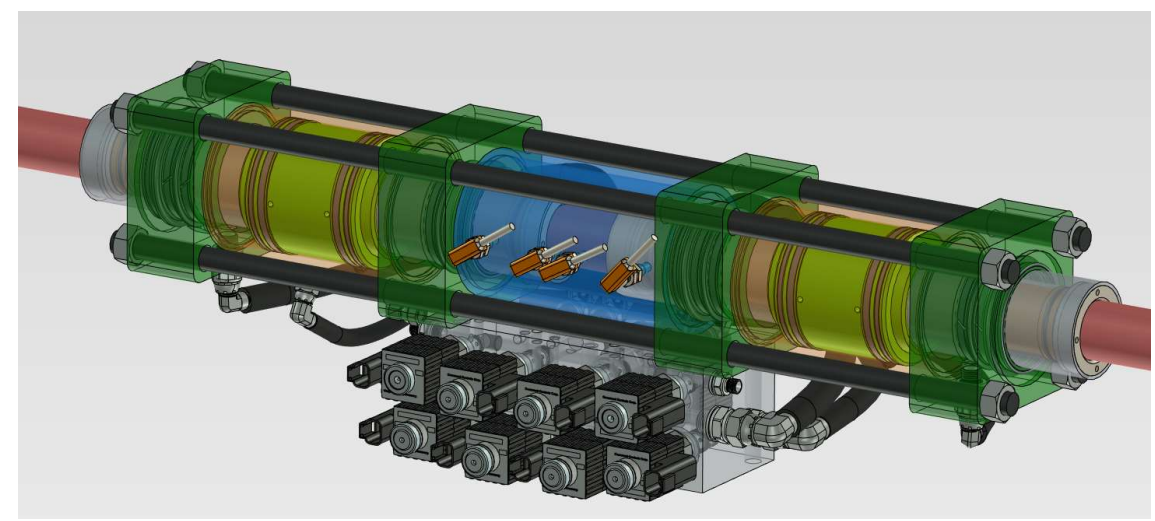

Figure 5. The HILA actuator. Illustration: Gustav Näslund, GN Tech.

\subsection{Issues with long linear motion with conventional hydraulic cylinders}

There are a few problems that occurs with long linear motion with conventional hydraulic cylinders. These are; buckling, hydraulic stiffness and hydraulic capacitance. Buckling is probably the main problem, limiting the stroke length of conventional hydraulic cylinders. It can be solved in some applications using a pulling cylinder instead.

Hydraulic stiffness and hydraulic capacitance affect the dynamic behavior and the controllability of the actuator system in an unfavorable manner. [4]. 
Long-stroke conventional hydraulic linear cylinders obviously have a number of difficult technical, economic and environmental problems. The advantages which normally are associated with the hydraulics; compactness and high power density diminish with increasing stroke length. The Hydraulic Infinite Linear Actuator (HILA) solves a number of the listed problem above. The HILA technology is characterized by providing very long strokes and high system pressure. The actuator has a higher stiffness compared to conventional hydraulic cylinders. Increased design pressure reduces the natural frequency of the system. These factors are favorable in actuator control design $[1]$.

When using long stroke lengths of hydraulic cylinders, it is usually difficult to use high hydraulic pressure as it reduces the natural frequency in the system. It can be shown that the natural resonance frequency $\omega_{h}$ for an elevator cylinder is:

$$
\omega_{\mathrm{h}} \propto \sqrt{\frac{\beta_{e}}{L_{\text {stroke }} * p_{\text {system }}}}
$$

where $L_{\text {stroke }}$ is the stroke of the cylinder and $p_{\text {system }}$ is the system pressure. Because the HILA cylinder has a much shorter stroke, a sufficiently high natural frequency can also be achieved at high system pressures [2]. However, the bulk modulus increases slightly with increasing pressure. The higher system pressure and small chamber volumes allows for an even more compact system design, with lower flow levels compared to low pressure systems, less energy losses in valves and a significant smaller reservoir volume.

Unique characteristics of the HILA actuator are the long strokes, the high system pressure and a compact and light actuator solution. It can be implemented in demanding environments, especially in mobile applications, where there presently are no alternative solutions. In contrast to ordinary ball screws or rack and pinion, HILA actuators also fit in applications used in tough environments, as forestry, agriculture and mining. In comparison with conventional long-stroke hydraulic cylinders, HILA can offer shorter, more compact and easier-to-place actuator solutions with long strokes. HILA is built on robust and cost-effective standard components. The technology is best suited for relatively slow movements and situations where the movement pattern is well known. The technology is patented.

Another advantage is that the system's need for hydraulic oil is only a fraction of what an ordinary hydraulic cylinder would require at the same stroke. Since the piston products in the cylinders are symmetrical. There is only marginal variation of the volume of oil in the system during feeding. The total volume of oil to minimal leakage of environmentally hazardous hydraulic oil into the vehicle.

Strokes of up to 7-8 $\mathrm{m}$ are possible depending on the piston rod diameter in horizontal applications, for pulling loads. Even longer strokes can be implemented in vertical applications, such as elevators. As the HILA technology needs a small cylinders and oil volumes is possible to design a very compact and long stroke electro-hydraulic actuator (EHA). The HILA technology also enables several active actuators to work on the same piston rod, which can be interesting for certain applications. This means that several processes can be performed in parallel instead of serially.

The disposition of the article is as follows. The first part is an introduction to the HILA technology and how heavy elements can be positioned with the technology. In the second part, it is showed how timber vehicles' aerodynamics can be significantly improved if stakes and banks are put together and a few other facilitating abilities for forestry logistics using HILA actuators. In the third part it is showed how agricultural can take advantage of the technology by flexibly position row units on a planter for optimal row distances from each other, to secure productive and sustainable farming.

\section{Need for linear positioning of heavy elements}

In some applications there is a need to position several heavy elements along a row, with high locking force either equidistant or with individual positioning. This need exists in several sectors, including agriculture, forestry, construction and logistics. For example, when you want to different bank and stakes for another load combinations (see fig. 6). 


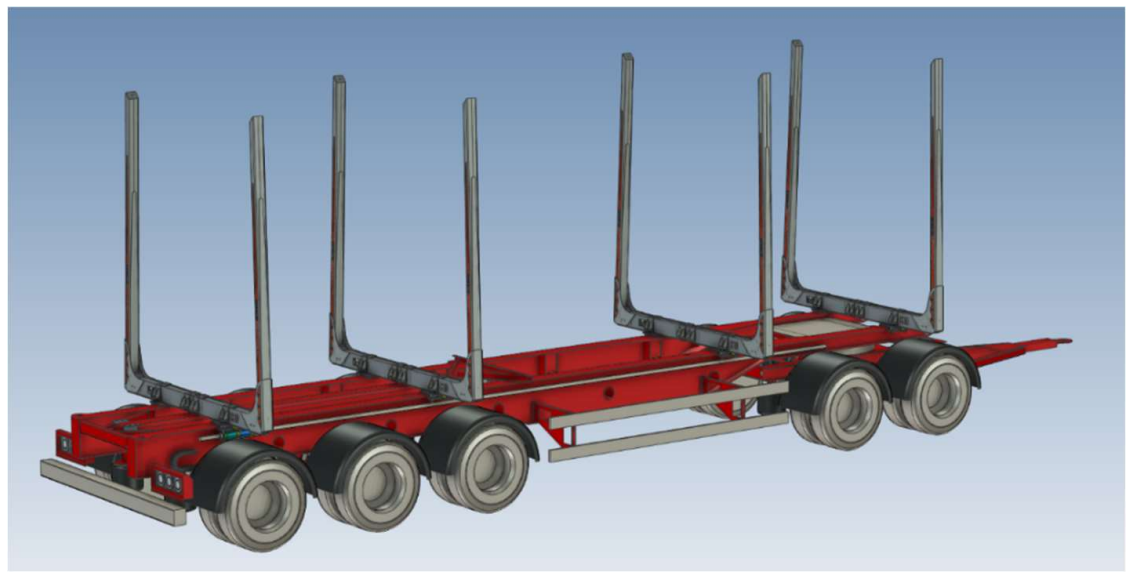

Figure 6. An unloaded timber trailer with banks and stake. Illustration: Gustav Näslund, GN Tech.

These elements need not be positioned by a dedicated actuator for each of the elements. Instead, a locomotivecarriage-like arrangement can be used, where the locomotive places the elements in the respective desired position. With the HILA actuator, several heavy elements can be placed and positioned in this desired way. These can be positioned with the actuator along the entire stroke. They are locked, for example, with a hydraulic clamping connection in the piston rod or in the surrounding fixed structure with an electromechanical lock.

One challenge, however, is to be able to position each element individually and in new patterns dynamically without having active locking mechanisms in each carriage element. An active locking mechanism that includes hydraulics or electromechanics means more potential fault modes compared to a passive solution, which has few possible fault modes.

If the distance between the elements in the extended position should always be the same, there is an easy way to access it with a passive method. By using fixed links between the elements, this need can easily be met. The weakness of this procedure means that manual intervention is required to change the length of the links. Another important aspect that must be considered is that when placing the elements along the linear path, they must be so attached to the fixed structure that drawer effects are avoided.

\subsection{Passive locking mechanism}

There is an easy way to create a passive locking mechanism. With a so-called friction coupling, the elements can be held in place. A friction joint is applied between the element and the linear path. The coefficient of friction is $\mu=0.4$. A suitable frictional force is applied to the joint which is large enough for the application to hold the element in place. This type of passive locking mechanism has been used with good results on timber banks on timber vehicles for many years. An element coming out of its dedicated position can be detected by a sensor located on the element and be repositioned.

\section{More efficient and greener timber trucks}

There is a great need in forestry transport to reduce fuel consumption in order to keep variable costs and carbon dioxide emissions down. Already 20 years ago, the issue of forestry transport and its contribution to carbon dioxide emissions in Sweden was lively debated. One solution was to develop vehicles that could take more load and thereby reduce both fuel consumption and carbon dioxide emissions in terms of transport work performed [5]. The expected fuel savings of about $20 \%$ could be met by a good margin. There are still opportunities to reduce fuel consumption that have not been studied in more detail, among other things by improving the vehicles' aerodynamics. Air and rolling resistance are two of the most important factors for fuel consumption.

Truck manufacturers put a lot of effort into reducing air resistance through better aerodynamic design of cab and chassis on towing vehicles with limited gains. For the trailers, no similar development has taken place. A timber vehicle, on the other hand, is like two completely different vehicles, loaded or unloaded. In unloaded condition, there are several points where turbulence occurs, not just behind the cab. Above all, around the vertical stakes, but also around the horizontal banks to which the stakes are attached, strong turbulence arises. Studying aerodynamic solutions that reduce air resistance for both loaded and unloaded timber vehicles has, as far as is known, never been tried before. 
Carried out wind tunnel studies show good opportunities to reduce air resistance [6]. Research is ongoing with the aim of developing, investigating and verifying the aerodynamic design of timber and wood chip cars that are heavier and longer than today's vehicles.

The simulation studies show that the biggest aerodynamic problems for unloaded timber trucks are partly the turbulence under the trailers that create air resistance, and partly stakes and banks that stand upright in the air stream. If these were bundled and assembled to one point, the air resistance would be significantly reduced. CFD (Computational Fluid Dynamics) simulations indicate reduced air resistance of around ten percent per measure, where the fuel savings can be estimated at 25-30 percent of the reduction in air resistance achieved, depending on the average vehicle speed. Today, there is no known technology or product on the market, for assembling banks and stakes on timber trucks [10].

There is a parallel to this with high aerodynamic resistance on unloaded timber train carriages. According to oral information an unloaded timber train gives a very large air resistance much due to the air turbulence that banks and stakes create [11]. Timber trucks may drive loaded and unloaded at $80 \mathrm{~km} / \mathrm{h}$ (Sweden) but freight trains may drive unloaded at $100-120 \mathrm{~km} / \mathrm{h}$. The air resistance increases with the square of the speed, ie it is also very interesting to put together banks and stakes even on unloaded timber trains. Studies on unloaded open coal wagons have shown that covering the wagons can reduce air resistance by $42 \%$ [7].

The trend for timber vehicles is towards longer distances and higher average speeds in Sweden. This is due to fewer and larger sawmills and pulp mills. As the air resistance increases with the square of the speed, the need for assembled banks and stakes for reduced fuel consumption further increases.

In other countries, such as the USA and Canada, unloaded timber vehicles are driven at a higher maximum speed, over $100 \mathrm{~km} / \mathrm{h}$, i.e. the benefits of assembled banks and stakes are even greater here. .

The bundling of banks and stakes on timber trains is also interesting, especially given the higher speeds of the unloaded timber trains. There are no technologies or products on the market for this today.

\subsection{Other needs in timber logistics}

In addition to lowering air resistance and fuel consumption on unloaded timber vehicles, there are several other needs to be met in timber logistics. It is also important to keep the empty weight of the timber vehicle down.

Most timber vehicles that load with their own crane have a bank shifter, a hydraulic cylinder that moves the banks for the trailer's rear stack forward to enable loading. The crane has a limited range. Then the whole stack is pushed back so that another stack can be loaded. The bank shifter is a $4 \mathrm{~m}$ long hydraulic cylinder that weighs $400 \mathrm{~kg}$. It also requires a large hydraulic tank. In addition, the bank shifter does not have sufficient stroke length to be able to assemble all the banks and stakes on a trailer. But with the compact HILA technology, it is feasible. The HILA system is estimated to weigh about $200 \mathrm{~kg}$, i.e. half of today's bank displacements and a smaller hydraulic tank can be used.

Today's timber transports are characterized by great variation in timber length. The length of the stacks can be changed between each timber transport. There is also a need to be able to combine different long stacks, from 2.6 to $6 \mathrm{~m}$ long stacks on the same timber vehicle. Today, this means a great deal of manual work to have to push the heavy banks and stakes between the various positions. Being able to operate everything from the truck cab with the push of a button on a display would significantly improve ergonomics for drivers and save a lot of time.

\subsection{HILA technology applied to timber trucks}

With the HILA technology, several heavy bank and stakes on a timber vehicle can be positioned individually with high locking force, that improves aerodynamics and reduces fuel consumption for heavy uunloaded timber vehicles. This is a major environmental benefit in terms of reduced emissions from fossil-fueled engines. With HILA technology, the vehicles' flexibility can significantly increase and open up for many more combinations that facilitate cost-effective and environmentally friendly logistics for both timber and complementary goods flow. HILA technology means that heavy and dangerous manual steps when changing banks and stakes on timber vehicles can eliminated. The adjustment can be performed from the cab via keystrokes. 
With the HILA actuator, the following functions can be implemented on towing vehicles and/or timber trailers (19):

1. Bundling of banks and stakes for better aerodynamics. The assembly of banks and stakes to one point on the trailer or behind the cab of the towing vehicle, will reduce air resistance, and fuel consumption and carbon dioxide emissions by about $5 \%$ [6], see fig. 7 .

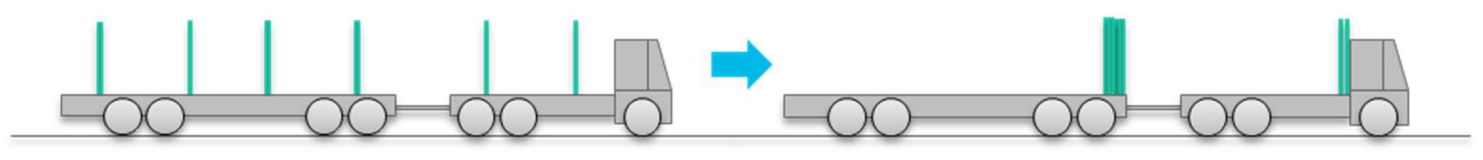

Figure 7. Bundling of banks and stakes

2. Bank shifting. Most timber vehicles that load with their own crane need to be able to move the rear stack of the trolley forward during loading. This bank shifting is done with a long stroke hydraulic cylinder. This can be solved just as well, but only with a predicted halved weight using the HILA actuator in comparison with today's bank shifters. This manoeuver needs a high actuator force, up to $170 \mathrm{kN}$.

3. Enable different bank and stakes combinations. The HILA actuator enables to switch to bank combinations for different lengths of wood (see fig. 8). The combinations are based on the number of stacks and the length of wood on each stack. This is especially important when the length of wood on a stack is to be changed from 2.6 to $6 \mathrm{~m}$. Timber trailers can have up to eight banks, which enables four stacks of short timber.

A large number of banks leads to higher air resistance and increased manual work when the banks are to be converted to fewer stacks. There are also risks in the working environment when this adjustment is done manually, especially in stressful situations.

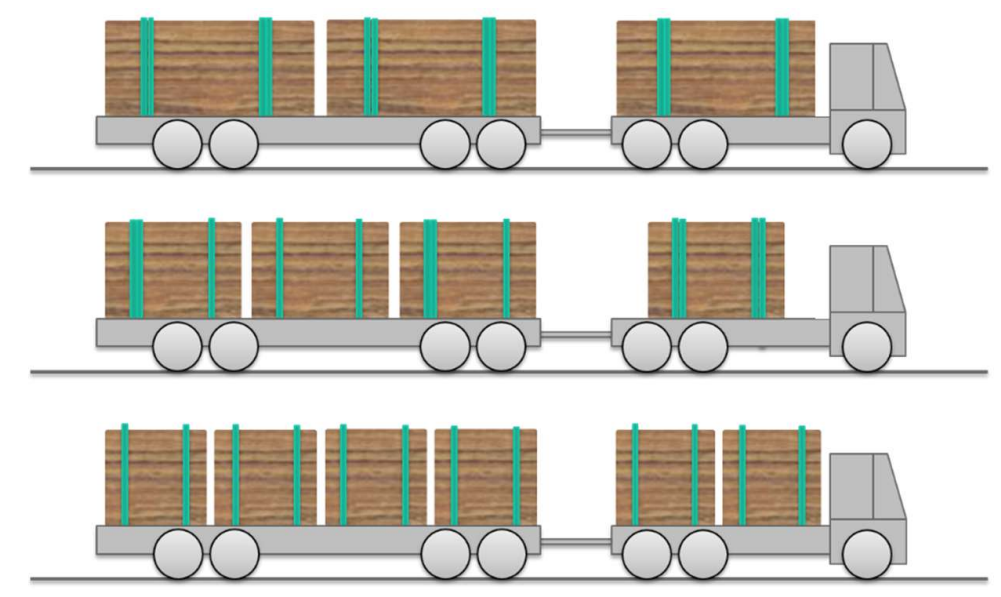

Figure 8. A timber vehicle with different load combinations.

With HILA actuator technology, adjustment between 2, 3 and 4 stacks combinations can be done smoothly simply with a push of a button in the cab. This saves time and creates a better working environment.

4. Adjustable center of gravity. Center of gravity balancing of stacks is important to get the right weight distribution and avoid overloading on any axis group.

5. Gap-filling. Minimizing gap between stacks, so-called gap filling, has two purposes; 1 . to reduce the air resistance of a loaded vehicle, and 2. to improve the torsional rigidity of the trailer. The latter can be done by pressing the two stacks of the trailer together so that they go into each other and partially hook together. 
6. Easier inspection. HILA can be used to disassemble stacks during inspection. For measurement of timber volume and quality control, it is important to have a certain distance between the stacks to be able to go in and measure with, for example, cameras [12].

7. Easier to turn. When banks and stakes are assembled in the middle of the trailer it will be easier to turn the vehicle in the woods. The risk of the stakes getting caught in trees and branches is thus reduced.

8. Allows for complementary cargo. Complementary freight flow for timber and container transport lead to savings with respect to time, costs and climate impact. With the HILA technology, towing vehicles and trailers can easily be rebuilt to carry $20^{\prime}-40^{\prime}$ containers (also applies to trains). This will create opportunities for valuable complementary freight flow and large savings with respect to time, costs and climate impact, especially on long transport routes (see fig. 9).

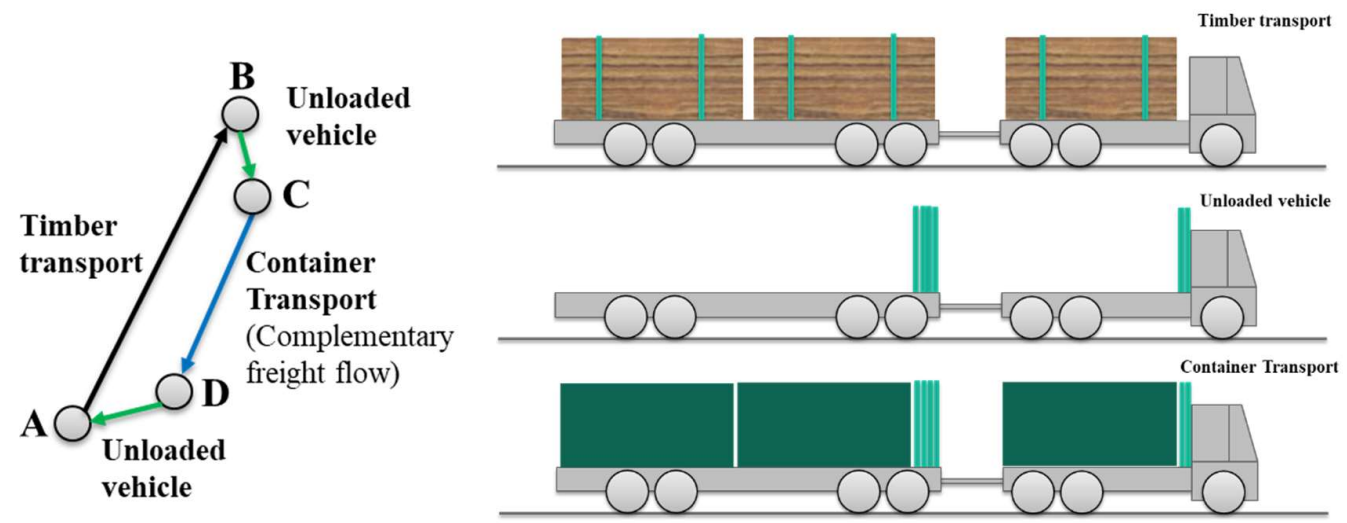

Figure 9. An example of a complementary freight flow schematic.

9. Use on timber trains. Bundling of banks and stakes on unloaded high speed timber trains can radically reduce air resistance and reduce energy consumption and in some cases also carbon dioxide emissions.

\section{HILA technology applied to precision seed drills}

Hydraulics has been important in agriculture since the 1940s and is still strategic. Today, hydraulics control a number of advanced functions. The hydraulics in turn are often controlled by advanced electronics in combination with sensors.

In the subject area of field machines in agriculture, since Jethro Tull's invention of the row seed drill around 1733, people have been interested in sowing, row cleaning and fertilizer filling etc. with different row distances [8]. Purely biologically, it is motivated to have different line spacings depending on crop or cultivation philosophy. But practical reasons have meant that you do not vary the distance in the way you would like due to lack of suitable actuators. Changing the row spacing is sometimes not even possible, and when possible, it takes time, which is rarely available and especially during spring sowing, as time is precious. Exceptions exist such as French Monosem [9], but their machine still has fixed positions and requires manual steps to change row spacing.

It can take two working days to manually rebuild a precision seed drill from 50 to $75 \mathrm{~cm}$ row spacing. This is a procedure that must be performed many times during a growing season by, for example, machine station owners. During the machine's lifetime of perhaps 20 years, the labor costs will exceed several 100,000s SEK. If the adjustment of line spacing can take place in a few minutes, this means large savings. Another way to solve the problem of variable row spacing today is to turn off certain row units so that 25,50 or $75 \mathrm{~cm}$ row spacing is easily obtained. But driving around with a very high dead weight, for example at a line distance of $75 \mathrm{~cm}$, is costly.

Fully flexible machines, however, still shine with some absence in the market, but there is a need for them. Another area of problems is how large wide carriages can be folded together to be transported on roads. Different countries have different rules for width, but usually the limit is 3 meters.

A key to secure productive and sustainable farming, is the placing of seeds at optimal distance from each other. However most, present seeders have fixed row distance and thus have low adaptability for different crops or soil conditions. With HILA technology, it is possible to implement a fast, efficient, and variable row spacing setting on machines for precision sowing, inter-row cultivation and crop harvesting. This means that the same machine can be used for different crops and meet farmers' demands for a common multi-purpose machine. 
HILA technology enables quick shift between different inter-row spacings simply from a display in the tractor cabin. It is possible to choose which crop to sow from a display in the tractor cabin and HILA sets the desired inter-row spacing automatically. The HILA concept allows infinite possibilities of configurations for different sort of seeds and the spacing can be independently adjusted with high precision. With the possibility of being able to vary the distance on the drill units, it means that the working width also varies. Thus, there is also the need to be able to vary the arm width of the machine. How this is solved is not described in this article. There are several capabilities of the HILA technology that can be integrated in a seed drill or planters.

With the HILA actuator, the following functions can be implemented on a seed drill:

1. Automatic change of row distance. It is possible to quickly vary the distance between drill units from a display in the tractor cab, i.e., without having to leave the cabin. This also includes the opportunity of fine-tuning the inter-row spacing for a certain crops and soils (see fig. 10).
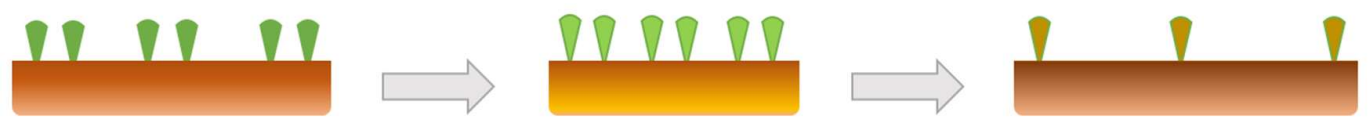

Figure 10. Different crops with different inter-row spacings.

2. Optimal center of gravity during folding. With HILA technology, it becomes practically possible to indirectly offer longer arms with a more optimal center of gravity during folding (see fig. 11). Another advantage is a simpler folding mechanism for changing 3-plex or 5-plex to 2-plex which gives opportunity to transport the row units a long distance. This also has potential to lower the folding time enabling faster moves between fields.

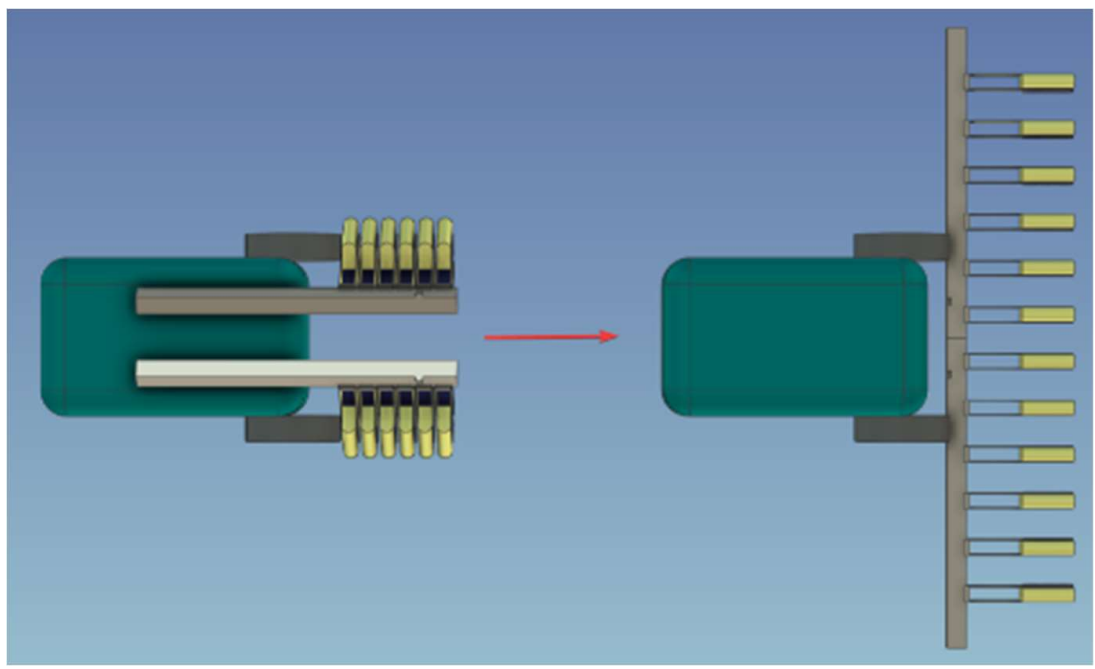

Figure 11. Unfolding the precision seed drill machine.

3. Optimal center of gravity during transport. With HILA it is possible to obtain a folding into a compact transport position, with the rows gathered with a low center of gravity of row units. This enables a more stable vehicle dynamics and that a higher speed can be allowed on the vehicle. This means a lot of valuable time saving when you must transfer between the fields. 
4. Reconfigure on the fly in case of row unit failure. Being able to quickly reconfigure if a row unit fails, means that planting can continue without having to wait for a service technician. This contributes to higher productivity (see fig. 12).

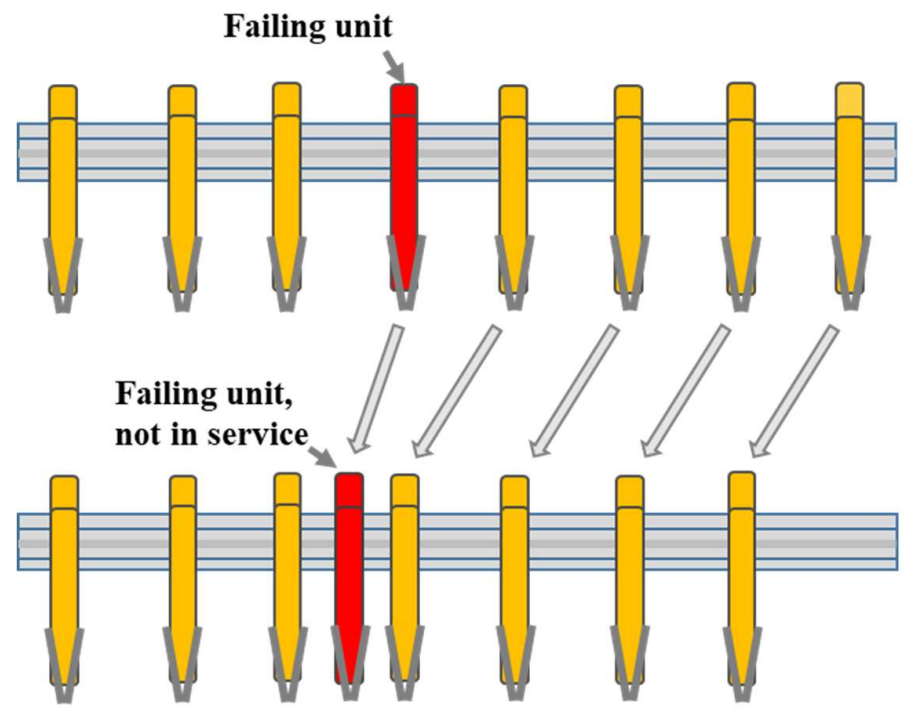

Figure 12. Reconfigure on the fly in case of row unit failure.

5. Reconfigure for service mode. During service or repair on a specific row unit, it is possible prepare large service space between the heavy row units, by pushing a button in the tractor cab (see fig. 13).
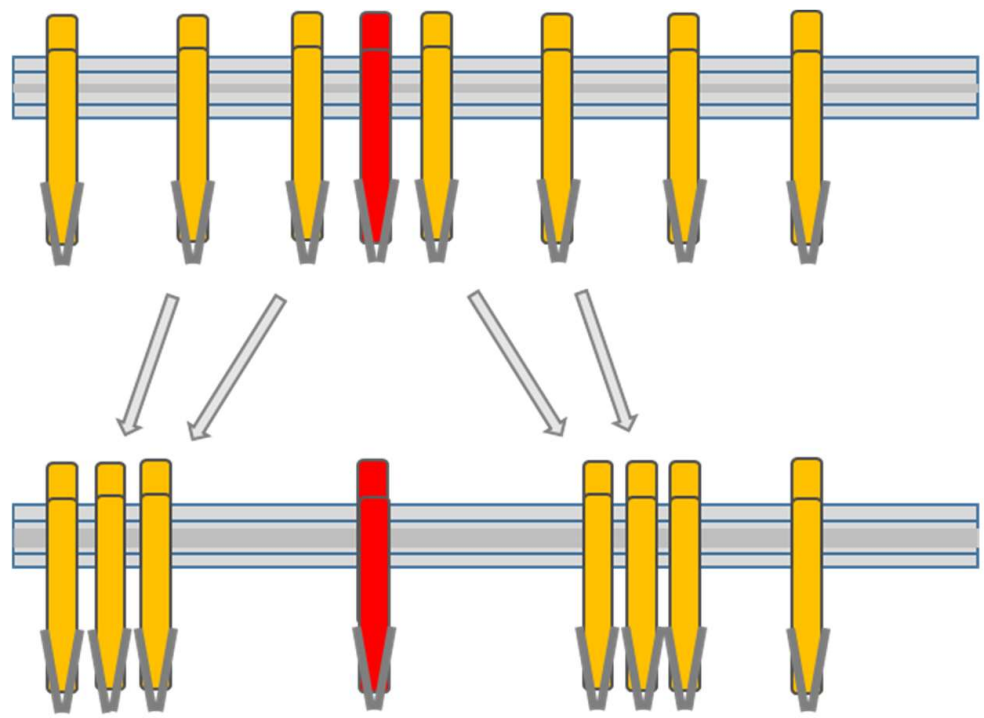

Figure 13. Reconfigure for service mode. 


\subsection{The HILA demonstrator}

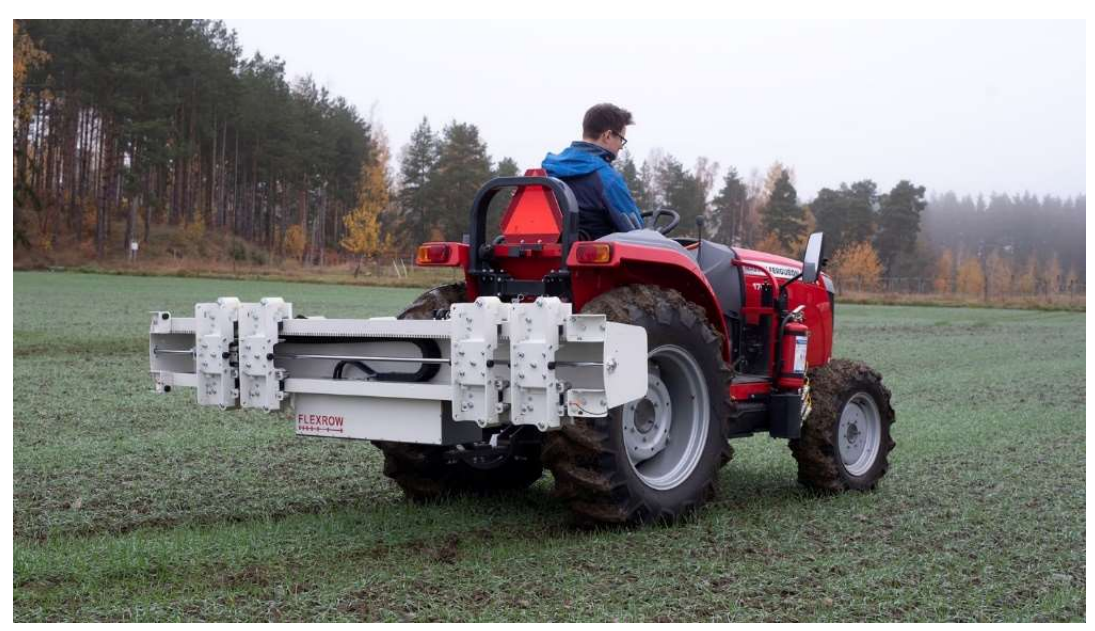

Figure 14. The HILA demonstrator ${ }^{1}$. Photo: Per Frankelius. LiU

A demonstrator of the concept has been developed with the aim that an actuator of this type can perform the most central task: the actuator can be connected to the row units and position them individually (see fig. 14). The demonstrator has been successfully tested and meets the initial requirements regarding functionality. The next step will be to build a full scale seed drill. The demonstrator is a result of a collaboration within the innovation program Agtech 2030. The collaboration has been conducted between Saab Ventures, The Rural Economy and Agricultural Societies (Hushållningssällskapet), Linköping University, Landberg Solutions and GN Tech. The hydraulic research division (FLUMES) at Linköping University has been instrumental for this work ${ }^{2}$.

\section{Discussion}

The HILA technology presented in this article shows several interesting applications in forestry and agricultural logistics. Several parallel development actions of HILA technology are underway in these sectors. In the forestry segment, a Vinnova-funded project is underway that aims to develop a first functioning HILA demonstrator for bundling banks and stakes that improves aerodynamics and reduces fuel consumption for heavy timber vehicles. In order to be economically viable, the chosen solution must also be able to completely replace the current bank shifting cylinder for load displacement. The project is primarily expected to provide a better understanding of the technology's functionality but also to get an indication of how energy consumption can be reduced for timber vehicles through better aerodynamics.

In the agricultural segment, a first demonstrator has been built and tested. It meets basic requirements for functionality. The actuator can be connected to the row units and position them individually. Subsequently, research has been carried out to gain a better understanding of the development needs and areas for improvement in sowing and mechanical weed control in agriculture and how HILA technology can improve current technology. The next step will be to develop a full-scale seed drill demonstrator based on this information. The purpose is to demonstrate the flexibility and robustness of HILA technology in the field.

Machine stations have a particularly large need to be able to offer different customers various row distances for sowing and mechanical weed control of different crops as well as quick transport between different fields. Semistructured interviews with farmers and experts have been performed. This research work has provided a lot of new knowledge and insights about different needs that exist today to be able to conduct a rational agriculture.

Based on this newfound knowledge, several interesting new possibilities and abilities that the HILA technology enables have been identified. One area that has particularly attracted extra interest is a rational control of weeds. It is becoming increasingly difficult to control weeds with chemical methods. More and more growers are choosing to switch to mechanical weed control. For example, there are great financial benefits from being able to go from manual weed cleaning to mechanical weed control for vegetable growers. Much of the economy of vegetable

\footnotetext{
${ }^{1}$ The commercial name of the HILA technology is Flexrow, see also www.flexrow.com.

${ }^{2}$ See film on https://www.youtube.com/watch?v $=$ cPK7-Wwx mk
} 
growing is determined by how effectively weed control can be carried out and how manual labor can be minimized. It is short periods of the year that sowing, and planting take place, respectively, row chopping. Everything happens under high time pressure. It must be easy and convenient to adjust the row spacing during inter-row cultivation.

When weeding in a row, there are two factors that are important to distinguish: partly the distance between the rows and partly the widths of row weeding. The technology for variable distance between the crop rows must be able to be combined with a variable width of the row harvester itself for it to be really effective. Different widths of row weeding are required for different times of the season. Field grown vegetables are weeded several times during the growing season and it requires narrower and narrower inter-row cultivation widths, as the crop grop grows.

It is important to enable inter-row cultivation as close to the crop as possible, in order to get the most out of the harvest. A key question in this context is how close to the crop you can do the inter-row cultivation without damaging the crop. That limit is dynamic, especially if there is large variation in how far the plants have come in their grooving. It often occurs that large and small plants are damaged when the row weeding cannot have a dynamic width. With modern positioning technology based on RTK / GPS, it is possible to implement variable row weeding width with one or a few centimeters at a time and thus be able to get closer to the crop.

The assessment is that with HILA technology, inter-row cultivation with variable row weeding width can be realized; to be able to quickly change the distance between the row cultivating bills and the width of the row cultivating bills. Furthermore, row spacing, and weeding width can be set with high accuracy and resolution.

An interesting possibility with HILA technology is to be able to switch between sowing units and units of interrow cultivation on the same machine. Thus, there are opportunities to obtain several capabilities in the same machine. Partly an efficient and flexible seed drill for different crops and partly an inter-row cultivation machine for different types of crops in different growing stages during the growing season.

\section{Conclusion}

In this paper a new hydraulic actuator technology has been presented, called HILA. HILA is based on a wellknown hydraulic clamping element technology, where the piston and the piston rod can be coupled and uncoupled by means of the clamping element. The innovation, in its simplest usage, provides new features to hydraulic cylinders, such as providing very long strokes and small chamber volumes, which means high stiffness and low capacitance. However, the invention also enables lower weight and volume of the actuator. The technology also represents a new sort of digital hydraulics. The technology is best suited for relatively slow dynamics and where the movement pattern is well-known in mobile hydraulic applications. The technology enables hydraulic actuators in new applications and expands field-of-use for hydraulic actuators, especially in the mobile domain.

The HILA technology enables several heavy elements to be positioned along a row, with high locking force either equidistant or with individual positioning. In this paper, two promising applications have been identified in timber logistics and agriculture that require positioning of several heavy elements. With this technology, specific problems within these domains can be solved in a completely new way. Forestry needs to develop transport vehicles with lower energy consumption. This can be done by improving the vehicles' aerodynamics. The air resistance of unloaded timber trucks and timber trains will be significantly reduced if stakes and banks are put together. They can be positioned individually with high locking force using the HILA actuator. In the agricultural context, it is possible to quickly change between different inter-row spacing when using HILA technology, enabling a multi-purpose seeder and mechanical weeding machine.

An agricultural demonstrator of the concept has been developed with the aim that an actuator of this type can perform the most central task: the actuator can be connected to the row units and position them individually. The demonstrator has been successfully tested and meets the initial requirements regarding functionality. The next step will be to build a full scale seed drill.

\section{Acknowledgement}

Research funding from Sweden's innovation agency Vinnova, the research and innovation program Agtech 2030 at Linköping University and Agtech Challenge at Rural Economy and Agricultural Societies has been platform for parts of the research behind this article. Some of the illustrations have been produced by Gustav Näslund at GN Tech AB. 


\section{References}

[1] Magnus Landberg, Martin Hochwallner, and Petter Krus. "Novel Linear Hydraulic Actuator”. ASME/BATH 2015 Symposium on Fluid Power and Motion Control (FPMC2015).

[2] Martin Hochwallner, Magnus Landberg, and Petter Krus. "The Hydraulic Infinite Linear Actuator properties relevant for control.” The 10th International Fluid Power Conference (10. IFK), 2016.

[3] ETP-OCTOPUS, ETP Transmission AB

[4] Magnus Landberg, Robert Braun, Magnus Sethson, and Petter Krus. "The Hydraulic Infinite Linear Actuator Applied to Elevators in Mid-Rise Buildings”. ASME/BATH 2018 Symposium on Fluid Power \& Motion Control (FPCM2018).

[5] Gunnar Svensson and Claes Löfroth, C. "ETT - Modulsystem för skogstransporter”, Skogforsk, Arbetsrapport 758, 2012

[6] Claes Löfroth and Olle Gelin. "A pilot study of aerodynamic design of forest vehicles", Arbetsrapport, Skogforsk nr. 870-2015, 2015

[7] Watkins, S., J. Saunders, and H. Kumar. “Aerodynamic resistance reduction of goods trains". Journal of Wind Engineering and Industrial Aerodynamics Volume 40 (2), p 147-178, 1992

[8] https://en.wikipedia.org/wiki/Jethro_Tull_(agriculturist) (retrieved 210520)

[9] https://www.monosem.com/News/News/Multi-purpose-MultiSlide-frame

[10] Oral reference Joakim Eriksson, ExTe, 2020

[11] Oral reference Owe Ljunghammer, ExTe, 2020

[12] www.cind.se/timber-industry (retrieved 210520) 